\title{
Pathology of Wild Strain (DP/As-Km/0019) of Duck Plague Virus Infection Revived in Ducklings
}

\author{
Mamta Pathak ${ }^{1 *}$, Shailesh Kumar Patel ${ }^{1}$, Syed Abdul Arif ${ }^{2}$, Nayanmoni Konwar ${ }^{3}$, Pallabi Pathak ${ }^{4}$ and \\ Naveen Kumar Verma ${ }^{5}$ \\ ${ }^{1}$ Division of Pathology, ICAR-Indian Veterinary Research Institute, Izatnagar, Bareilly, Uttar Pradesh, INDIA \\ ${ }^{2}$ Department of Veterinary Clinical Medicine, Ethics and Jurisprudence, College of Veterinary Science, \\ Assam Agricultural University, Khanapara, Guwahati, INDIA \\ ${ }^{3}$ Department of Veterinary Microbiology, College of Veterinary Science, Assam Agricultural University, Khanapara, \\ Guwahati, INDIA \\ ${ }^{4}$ Department of Veterinary Parasitology, College of Veterinary Science, Assam Agricultural University, Khanapara, \\ Guwahati, INDIA \\ ${ }^{5}$ Division of Surgery, ICAR-Indian Veterinary Research Institute, Izatnagar, Bareilly, Uttar Pradesh, INDIA
}

"Corresponding author: M Pathak; E-mail:mamtapathak48@gmail.com

Received: 23 July, 2019

Revised: 16 Oct., 2019

Accepted: 20 Oct., 2019

\begin{abstract}
Duck plague is an acute highly contagious disease of duck, geese and swan of all ages caused by Anatid Herpesvirus-1. The disease is characterized by significant decrease in growth, egg production and sudden death along with high morbidity and mortality which results into significant economic losses in duck industry. In present study, the pathology of wild strain (DP/ As-Km/0019) of duck plague virus (DPV) was experimentally studied in 2 months old ducklings. The prominent clinical signs observed were depression, loss of appetite, greenish diarrhoea, ruffled feathers, nasal discharge, lacrimation and pasted eyelids with periorbital ring formation. The significant gross lesions were observed in spleen, oesophagus, liver, heart, brain and intestine. Vascular and degenerative changes like congestion, haemorrhages, necrosis and diphtheritic membrane formation were noticed in various parenchymatous organs. Microscopically, focal to diffuse areas of haemorrhages, coagulative necrosis, and fatty changes in liver, focal emphysema in lungs, formation of diphtheritic membrane on the mucosal surface along with presence of numerous infiltrating cells in oesophagus were recorded. Presence of Anatid Herpesvirus-1 viral DNA in liver and spleen tissue samples was detected by PCR.
\end{abstract}

Keywords: Pathology, Duckling, Assam, Duck Plague

Among the various types of livestock farming, duck farming plays an important role in the socio-economic development of the farmers. Duck farming is a highly profitable industry because of its high prolificacy and adaptability to free range system of rearing. As per $19^{\text {th }}$ Livestock Census (2012), the total duck population of India is 23.54 million and Assam ranks first with 7.31 million ducks. Duck plague was first reported from West Bengal (Mukherji et al., 1963) in India.

Duck Plague is an acute highly contagious and infectious disease caused by Anatid herpes virus-1, a member of the
family-Herpesviridae, subfamily-Alphaherpesvirinae. The disease is characterized by severe diarrhoea, sudden death, vascular damage, internal haemorrhages, lymphoid depletion, mucosal eruptions and degenerative lesions in almost all the parenchymatous organs (Dhama et al., 2017). Although mortality and morbidity depends upon the virulence of the virus and immunological status of the

How to cite this article: Pathak, M., Patel, S.K., Arif, S.A. Konwar, N., Pathak, P. and Verma, N.K. (2019). Pathology of wild strain (DP/As$\mathrm{Km} / 0019)$ of duck plague virus infection revived in ducklings. J. Anim. Res., 9(6): 831-835. 
host (Goldberg et al., 1990), ducks of all age groups are considered susceptible with highest mortality in ducklings. Under natural conditions, ducks are usually infected through close contact to infected ones (Kaleta et al., 2007). The virus replicates principally in the epithelial cells and lymphocytes (Shawky, 2000; Yuan et al., 2005). Clinical signs like partially closed eyelids with photophobia, anorexia, extreme thirst, ataxia, oculo-nasal discharge, and greenish diarrhoea are characteristic to the disease (Dhama et al., 2017; Konch et al., 2009). Post-mortem lesions viz. petechiae over the epicardium, presence of the diphtheritic membrane over the esophageal folds and haemorrhages in intestine, spleen, liver, kidney, lungs, payer's patches and bursa of Fabricius are consistent findings of the disease (Konch et al., 2009; Doley et al., 2013).

In spite of taking all important measures for preventing the disease, it is still prevalent in the country and threatening the existence of duck population and survivability of the duck farmers in particular. In present study, the pathology of wild strain (DP/As-Km/0019) of duck plague virus (DPV) was experimentally studied in 2 months old ducklings in order to understand the pathology and pathogenesis of the virus.

\section{MATERIALS AND METHODS}

This study was carried out in the Department of Pathology and Department of Microbiology, College of Veterinary Science, Assam Agricultural University, Khanapara, Guwahati.

\section{Virus}

DPV (DP/AS-KM/0019) positive tissues obtained from the repository of the Department of Microbiology were used for inoculation of ducklings in order to study the pathology of the virus.

\section{Ducklings}

A total of 12 ducklings of 2 months age were procured from backyard duck rearing areas of Assam. Ducklings were screened for presence of maternal antibody against DPV using indirect ELISA (I-ELISA). The ducklings were divided into 2 groups with 6 ducklings in each group, one group was used for inoculation and the other was kept as control. The ducklings were maintained using good managerial practice and under keen observation in the Department of Veterinary Pathology, College of Veterinary Science, Assam Agricultural University, Khanapara, Guwahati.

\section{Screening of ducklings for DPV specific antibody by I-ELISA}

The ducklings were screened for maternal antibody by I-ELISA as per the method described by Morrissy et al. (2004). A chequer board titration was performed for optimization of working dilution of DPV antigen and antibodies as per standard protocol.

\section{Preparation of inoculum}

PCR positive post-mortem samples (liver and spleen) obtained from repository of Department of Veterinary Microbiology was used to prepare the inoculum. The samples were grounded in a mortar-pestle into a $20 \%$ suspension (weight/volume) in sterile PBS. The suspension was then centrifuged at $3000 \mathrm{rpm}$ for 10 minutes and the supernatant was collected, this step was repeated thrice. The final supernatant was subjected to cocktail antibiotic treatment for 45-60 minutes and used as inoculum.

\section{Inoculation of DPV in ducklings}

The inoculum was inoculated @ 1ml/duckling via oral $(0.5$ $\mathrm{ml})$ and intramuscular $(0.5 \mathrm{ml})$ route, whereas in control group sterile PBS was inoculated via same route and with same dose. Ducklings were observed for the development of post inoculation signs and were sacrificed on $7^{\text {th }}$ day of post infection. Gross changes present in the different internal organs were recorded and simultaneously tissue samples from liver, spleen, kidney, oesophagus, lungs and heart were collected in 10\% neutral buffered formalin for histopathology and on ice for molecular confirmation by PCR.

\section{Molecular studies}

Pooled post-mortem tissue samples were homogenized and $20 \%$ suspension was prepared by using appropriate volume of PBS ( $\mathrm{pH}$ 7.4). Extraction of viral DNA from the inoculum, post-mortem tissues, tracheal and cloacal swabs 
preserved in HBSS and whole blood was done by Trizol method as per manufacturer's recommendations. The primer pairs, F-5' GAAGGCGGGTATGTAATGTA-3' and R-5' CAAGGCTCTATTCGGTAATG-3' were used for amplification of DPV DNA (UL30 gene) (OIE, 2012).

\section{Histopathology}

Representative samples from all the organs were further subjected for the histopathological examination. Dehydration and clearing was done by using alcohol and xylene followed by wax impregnation and sectioning at $4 \mu$ thickness. Hematoxylin and eosin staining was done as per standard procedure (Luna, 1968).

\section{RESULTS AND DISCUSSION}

The ducklings used in the study were found free from maternal antibody. Presence of viral nucleic acid, in the inoculum used in the study and pooled tissue samples of experimental ducklings was detected by PCR. The yield of DNA was ranged between 85 to $750 \mu \mathrm{g} / \mathrm{ml}$. Test spleen and liver samples showed the amplicon size of $446 \mathrm{bp}$ and no amplification was observed in positive control group (Fig. 1).

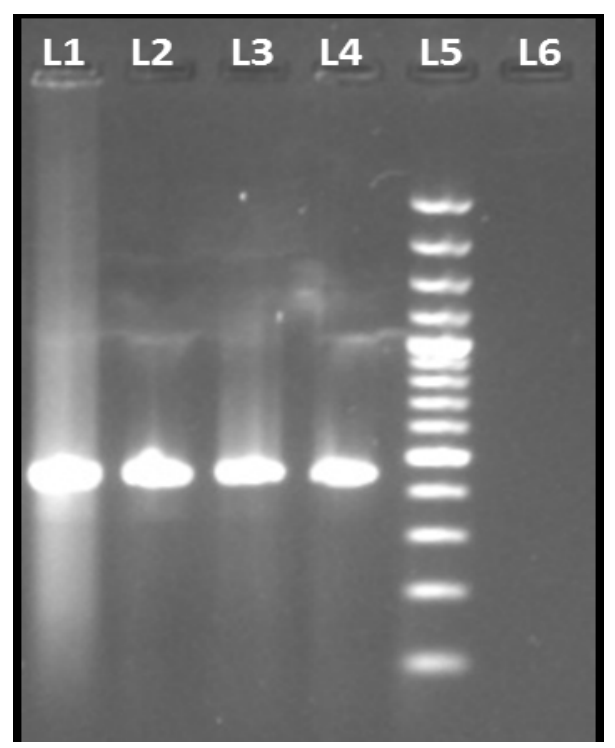

Fig. 1: PCR products in ethidium bromide stained 1.7\% agarose gel electrophoresis. L1: Inoculum used for the experiment; L2: Pooled spleen samples of infected ducklings; L3: Pooled liver samples of infected ducklings; L4: Positive control; L5: $1 \mathrm{~kb}$ DNA ladder and L6: Negative Control.
Pathology of DPV in ducklings was previously reported by Bhowmik and Chakrabarty (1985) and El-Samadony et al. (2013). They reported that duckling was unable to stand with anoraxia, diarrhea and death. The gross lesions are characterized by vascular damage with tissue haemorrages. Lesions of the lymphoid tissues were more prominent than visceral haemorrages. Present study revealed good revival of wild strain of DPV in ducklings inoculated with PCR positive tissue samples which indicates well adaptation of virus to primary host. In order to recover DPV isolate from field tissue samples, duckling can be an ideal in vivo host. In context to this, Sarmah (2016) found similar result with revival of the wild strain of DPV in the primary host.

The infected ducklings showed clinical signs between 4 to 7 days of post inoculation (dpi). The clinical signs were noticed as early as 3 dpi onwards. The common clinical signs observed in most of the ailing ducklings were depression, lethargy, huddling, unapparent to the environment, reduced appetite followed by anorexia noticed from $4 \mathrm{dpi}$, increased thirst, nasal discharge, ruffled feathers, periorbital ring formation with lacrimation, pasting of feathers around the eyes, greenish diarrhea with soiled vent, ataxia and dropping of wings (Fig. 2). Our findings were closely related with the findings of Konch et al. (2009) and Sahariah (2016).

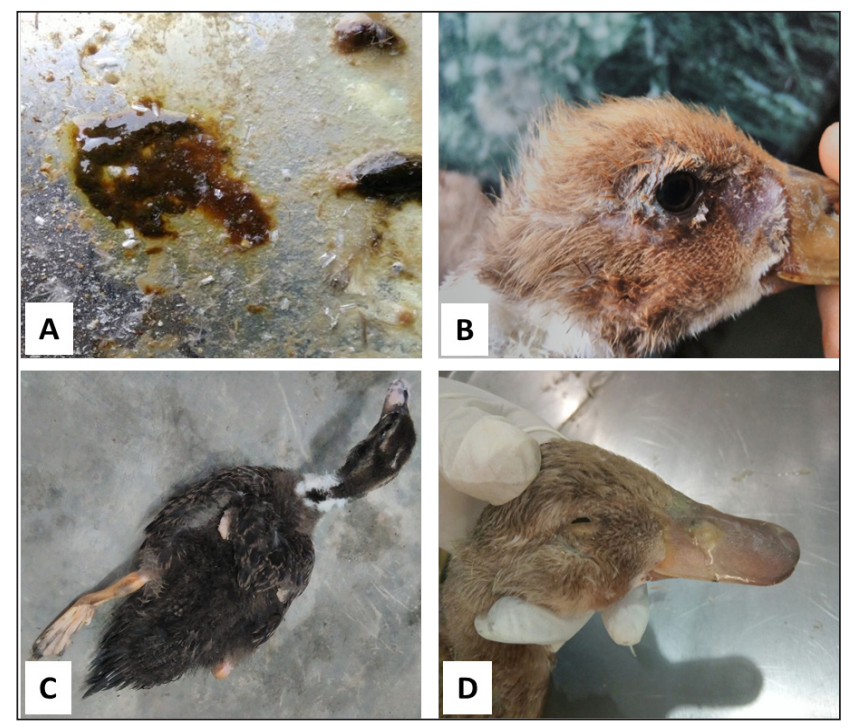

Fig. 2: Clinical signs showing by duck plaque infected ducklings. A: Greenish diarrhoea of ailing duck; B: Duckling infected with DPV exhibit periorbital ring formation with lacrimation; C: Duckling showing paralysis of left limb; D: Catarrhal nasal discharge showing by infected ducklings 
The most characteristic gross lesion observed was deposition of yellowish diphtheritic membrane along the longitudinal folds of oesophagus. The lesion was focal and limited to certain areas of oesophagus moreover; removal of the plaques did not reveal any haemorrhagic areas. Moderate catarrhal enteritis with absence of gross lesions in annular band was evident. Grossly, almost all affected birds showed - hepatomegaly, focal petechial hemorrhages over the liver parenchyma and gall bladder distended with bile. Heart showed congestion of blood vessels along with a few focal petechial haemorrhages over the epicardium. In kidneys, diffuse petechial haemorrhages with few necrotic foci over the parenchyma were observed. Lung parenchyma revealed emphysema associated with diffuse haemorrhage. The spleen was dark in colour and focal areas of haemorrhages were evident over the parenchyma, however splenomegaly with severe congestion was the regular finding in some of the infected birds. The mucosa of the trachea was congested with mild haemorrhages. . Brain revealed mild to moderate congestion of meningeal blood vessels. (Fig. 3). Gross lesions were mainly confined to spleen, oesophagus, liver, heart, lungs, kidney, trachea and brain. These above lesions were in accordance with the findings of Dhama et al. (2017).

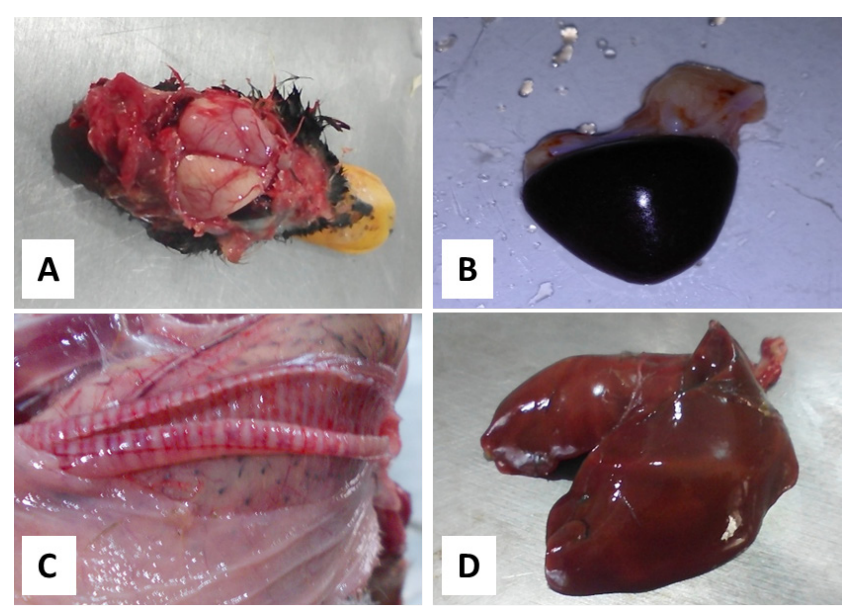

Fig. 3: Gross lesions in various organs of infected ducklings. A: Brain showing meningeal congestion; B: Enlarged and severely congested spleen; $\mathbf{C}$ : Trachea showing severe haemorrhages and congestion; D: Liver showing hepatomegaly with congestion and hemorrhages.

Microscopically, sloughing of diphtheritic membrane from the mucosal surface and infiltration of numerous mononuclear cells were evident in the oesophagus. Liver showed haemorrhages, disintegration and coagulative necrosis of hepatic cords along with necrosis of hepatocytes. In heart, haemorrhages between the muscle fibers were observed. Kidneys were characterized by congestion and haemorrhages in interstitial space, shrinkage of glomerular tuft, increased Bowman's space, coagulative necrosis and desquamation of renal tubular epithelium. In lungs, emphysema associated with diffuse haemorrhages was observed. Depletion of lymphocytes in lymphoid follicles and diffuse areas of haemorrhages were observed in spleen (Fig. 4). Histopathologically, most consistent lesions were observed in liver, spleen, oesophagus, heart, lungs and kidneys in the present study and similar findings were also reported by Shawky (2000) and Sahariah (2016).

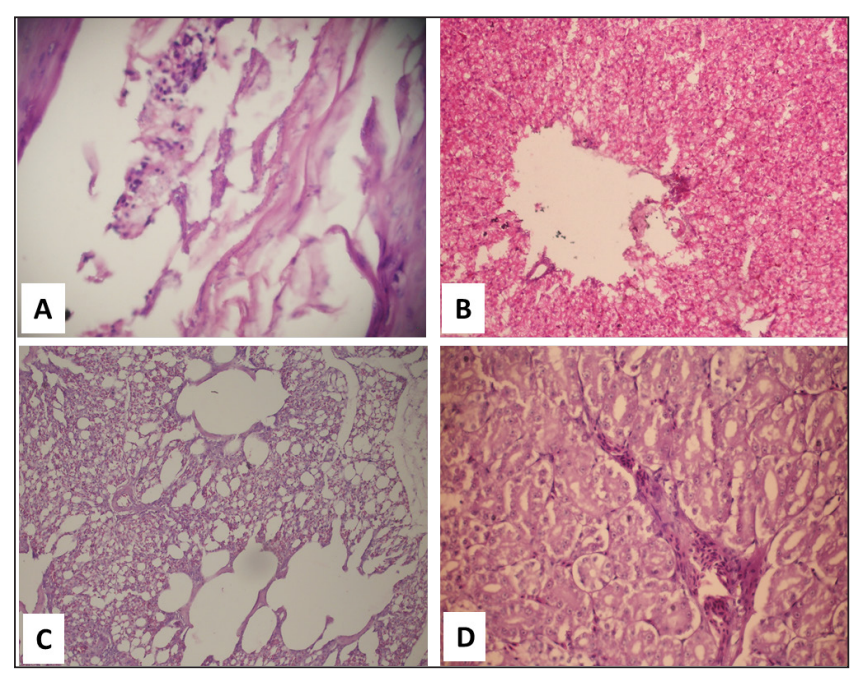

Fig. 4: Microscopic lesions in various organs of duck plague infected ducklings. A: Oesophagus showing sloughing of oesophageal mucosa with presence of mononuclear inflammatory cells $(\mathrm{H} \& \mathrm{E} \times 400)$; $\mathbf{B}$ : Disintegration and degeneration of hepatic cords along with haemorrhages and coagulative necrosis of hepatocytes $(\mathrm{H} \& \mathrm{E} \times 100)$; C: Lungs showing focal emphysema with diffuse haemorrhages $(H \& E \times 100)$; D: Congestion of renal blood vessels with severe tubular degeneration in kidney (H\&E $\times 400)$.

\section{CONCLUSION}

The present study, concluded that wild strain of DPV present in post-mortem tissue samples could be revived in the primary host. Periorbital ring formation was the constant clinical finding in experimentally infected ducklings. Formation of diphtheritic membrane in the 
oesophagus, haemorrhages along with necrosis in liver and depletion of lymphocytes in splenic follicles are the major gross pathological findings. Microscopically, the most prominent changes were characterized by congestion, haemorrhages, degeneration and necrosis of the parenchymatous organs.

\section{REFERENCES}

19 ${ }^{\text {th }}$ Livestock Census. 2012. All India Report, Ministry of Agriculture, Department of Animal Husbandry, Dairying and Fisheries, Krishi Bhawan, New Delhi.

Bhowmik, M.K. and Chakrabarty A.K. 1985. Occurrence of duck plague in ducklings of West Bengal. Indian. J. Poult. Sci., 20: 114-118.

Dhama, K., Kumar, N., Saminathan, M., Tiwari, R., Karthik, K., Kumar, M., Palanivelu, M., Zubair, M., Malik, Y. and Singh, R. 2017. Duck virus enteritis (Duck Plague) - a comprehensive update. Vet. Q., 37(1): 57-80.

Doley, M., Das, S.K., Barman, N.N. and Rajbongshi, G. 2013. Adaptation of vaccine strain of duck plague virus in chicken embryo fibroblast cell culture. Indian. J. Anim. Sci., 83(9): 880-882.

El-Samadony, H.A., Tantawy, L.A., Salama, E. and Khedr, A.A. 2013. Molecular characterization of circulating duck viral enteritis in Egypt during 2012-2013. Br. J. Poult. Sci., 2(3): $38-44$.

Goldberg, D.R., Yuill, T.M. and Burgees, E.C. 1990. Mortality from duck plague virus in immunosuppressed adult mallard ducks. J. Wild. Dis., 26(3): 299-306.

Kaleta, E.F., Kuczka, A., Kuhnhold, A., Bunzenthal, C., Bonner, B.M., Hanka, K., Redmann, T. and Yilmaz, A. 2007. Outbreak of duck plague (duck herpes virus enteritis) in numerous species of captive ducks and geese in temporal conjunction with enforced biosecurity (in-house keeping) due to the threat of avian influenza A virus of the subtype Asia H5N1. Dtsch. Tierarztl. Wochenschr., 114(3): 3-11.
Konch, C., Upadhyaya, T.N., Goswami, S. and Dutta, B. 2009. Studies on the incidence and pathology of naturally occurring duck plague in Assam. Indian. J. Vet. Pathol., 33(2): 213-215.

Luna, L.G. 1968. Manual of histologic staining methods of the Armed Forces Institute of Pathology. Third edition, the Blakiston Division, McGraw-Hill Book Company.

Morrissy, C.J., Daniels, S.L., Lowther, S.L., Goff, W., Pritchard, I., Tu, T.D., Phuc, K.V., Hung, D., Hong, N.T.L., Trung, N.T., Spradbrow, P.B.M. and Westbury, H.A. 2004. Duck plague in Vietnam and the development of diagnostic capability. ACIAR Proc., No.117.

Mukherji, A., Das, M.S., Ghosh, B. and Ganguly, J.L. 1963. Duck plague in West Bengal. Ind. Vet. J., 40: 457-462.

OIE Terrestial Manual. 2012. Duck virus enteritis, Chapter 2.03.07. (https://test.oie.int/fileadmin/Home/eng/Health_ standards/tahm/2.03.07 DVE.pdf)

Sahariah, P.J. 2016. Studies on the prevalence, pathology and molecular diagnosis of duck virus enteritis in Assam. M.V.Sc. Thesis Assam Agricultural University, Khanapara, Guwahati.

Sarmah, H. 2016. Molecular and biological characterization of wild strains of duck plague virus. M.V.Sc. Thesis, Assam Agricultural University, Khanapara, Guwahati.

Shawky, S. 2000. Target cells for duck enteritis virus in lymphoid organs. Avian. Pathol., 29: 609-616.

Yuan, G.P., Cheng, A.C., Wang, M.S., Liu, F., Han, X.F., Liao, Y.H. and $\mathrm{Xu}, \mathrm{C} .2005$. Electron microscopic studies of the morphogenesis of duck enteritis virus. Avian. Dis., 49: 50-55. 
